\title{
Target validation using chemical probes
}

\author{
Mark E Bunnage, Eugene L Piatnitski Chekler \& Lyn H Jones
}

\section{Fully profiled chemical probes are essential to support the unbiased interpretation of biological experiments necessary for rigorous preclinical target validation. We believe that by developing a 'chemical probe tool kit', and a framework for its use, chemical biology can have a more central role in identifying targets of potential relevance to disease, avoiding many of the biases that complicate target validation as practiced currently.}

M edicinal chemistry design and synthesis can provide selective tool compounds to interrogate biology, thus illustrating the synergy between chemical biology and drug discovery ${ }^{1,2}$. A major issue with using small molecules to augment target validation before launching a full drug discovery program is having the confidence that we have effectively validated the target of interest in a relevant phenotypic assay. There are many widely used chemical probes that do not meet generally accepted potency and selection criteria, and the conclusions made from their use are suspect. Similarly, the use of selective chemical probes in heavily manipulated biological assays is less likely to generate information of relevance to human disease.

When considering this problem, we can directly draw from our experiences in clinical drug development. A retrospective analysis of 44 drug programs in phase 2 clinical trials at Pfizer revealed that most failures resulted from a lack of efficacy, whereas the successful programs achieved what is termed the 'three pillars of survival': pillar 1, sufficient exposure at the site of action; pillar 2, proof of target engagement; and pillar 3, expression of functional pharmacological activity ${ }^{3}$. Moreover, for the

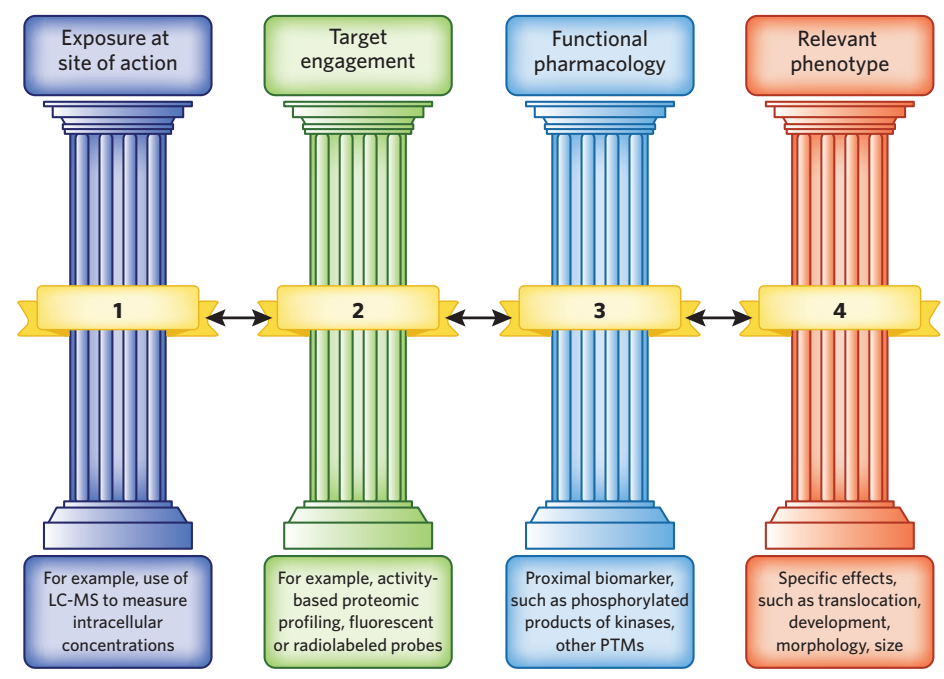

Figure 1 | The four pillars of cell-based target validation using chemical probes.

\section{Box 1 | The four pillars of target validation.}

Pillar 1. Exposure at the site of action. Experiments should confirm that the probe is able to achieve pharmacologically relevant concentrations inside or at the cell. 'Mismatches' between the biochemical activity of a probe (for example, inhibition of recombinant enzyme activity) and whole-cell activity are often explained by invoking poor cell permeability, but usually there are no data to confirm this hypothesis. Analytical techniques can be used to measure intracellular (and possibly subcellular) concentrations of a probe.

Pillar 2. Target engagement. This is the most technically challenging pillar, though it is essential to link exposure at the site of action (pillar 1) to pharmacology (pillar 3) and phenotype (pillar 4). Exciting advances in techniques such as activity-based proteomics have enabled the quantification of target engagement to be made. Sophisticated functional probes can measure occupancy inside the cell and facilitate unbiased selectivity determination in a more physiologically relevant environment.

Pillar 3. Expression of functional pharmacology. Assays can be created that measure the pharmacology of the probe, often assessing a proximal biomarker for activity, for example, the phosphorylated product of a kinase, an acetylated histone tail or depletion of an HSP9O client protein.

Pillar 4. Proof of phenotype perturbation. The challenge for cell biology is to create assays that capture the most relevant phenotypic changes in the context of human disease and for which there is a high degree of confidence in their 'translatability'. Phenomena that may lead to false positive results, such as nonspecific cell death, should be ruled out at an early stage. The strongest rationale for target validation can be provided if all four pillars are built in the same pathophysiologically relevant cell system. 


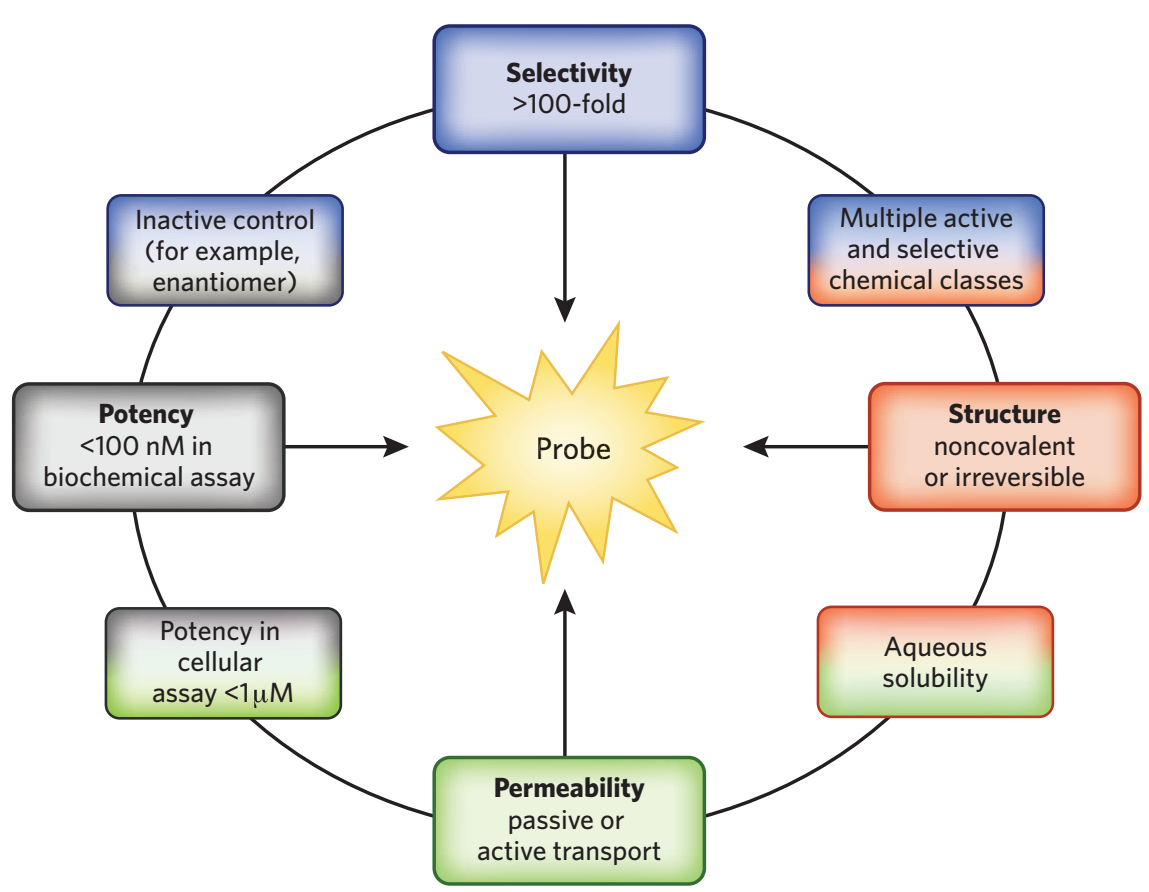

Figure $\mathbf{2}$ | Important criteria for designing and creating effective chemical probes. Selectivity against close-family-member biological targets, chemical structure and mode of inhibition, cell permeability and biochemical activity are four essential considerations in designing successful chemical probes. Additional considerations may include aqueous solubility and potency in cellular assays, the value of building a chemical tool kit that includes probes from orthogonal active and selective chemical classes and the importance of having structurally related inactive controls (for example, an inactive enantiomer, if available). Threshold values for potency and selectivity are discussed in the literature ${ }^{2}$.

projects that failed, it was often impossible to ascertain whether the target had been effectively tested in the clinic owing to gaps in some or all of the three pillars, reinforcing the need for an integrated understanding of the pharmacokinetic and pharmacodynamic characteristics of a drug to be established ${ }^{4}$.

Here we suggest that the three pillars concept provides a framework that may also be useful in the exploratory process of cellbased target validation using chemical probes, with the addition of a 'fourth pillar': proof of phenotype perturbation (Fig. 1 and Box 1 ).

By applying the principles of the four pillars to the use of chemical probes in cell-based assays, we propose that a more confident link between target perturbation and disease-relevant pharmacological modulation might be made. Here, we highlight examples of enabling technologies that can be applied in this context and provide an example of the successful application of the four pillars philosophy.

\section{Pillar 1: cellular penetration}

The characteristics for quality smallmolecule probes have been reviewed previously ${ }^{2}$, and an outline of the key attributes is included here (Fig. 2). Cell permeability is a prerequisite for the assessment of phenotypic effects of a small-molecule probe in whole cells, and confirming that the probe can access the site of action (pillar 1) is therefore essential. Validating probe accessibility is particularly important in ruling out false negatives, which can occur when a molecule's inability to enter a cell results in the lack of a pharmacological (pillar 3) or phenotypic (pillar 4) response. Physicochemical requirements of chemical probes have been delineated ${ }^{2}$ (Fig. 2), and just as Lipinski's 'rule of five' is used to understand the absorption of drugs ${ }^{5}$, certain criteria have been established to improve cellular penetration of a chemical compound. For example, tactics such as increasing lipophilicity of a probe can be used to improve both potency and membrane permeability; however, this may negatively affect aqueous solubility and selectivity.

Additionally, it may be necessary to measure the concentration of probes inside the cell to accurately determine dose responses and structure-activity relationships (SARs). Scientists often assume that the concentration of the compound added to a cell is equal to the concentration inside the cell, but this need not necessarily be the case, particularly if there is limited cellular penetration or active efflux or, conversely, if active uptake and accumulation occurs.
Another related aspect of pillar 1 is ensuring that exposures are commensurate with the on-target activity of the probe and not in great excess, such that selectivity windows over off-targets are eroded. This is important as the use of probes in cell biology experiments at concentrations in excess of their selectivity window could lead to erroneous links being made between on-target activity and phenotypes, when it may be the off-target activity that is actually driving the observed response. We would recommend this as a key consideration in target validation studies, even when using quality probes with high selectivity.

Simple techniques can be applied to measure in-cell concentrations, such as LC-MS analysis of cell extracts. Local concentrations in specific subcellular compartments may also vary (subcellular compartments can even be targeted in the chemical probe design), and therefore more stringent fractionation might be required, together with the application of sophisticated microscopic imaging, MS and radiometric methods of detection and quantification ${ }^{6}$. An accurate and quantifiable pillar 2 (measurement of target engagement inside the cell) also, by definition, provides pillar 1 .

Pillar 2: target engagement and selectivity Simple biochemical assays, though useful to drive the drug discovery process through optimization of potency and selectivity, provide limited information regarding the performance of a chemical probe in a whole-cell or in vivo context. For cell biology applications, it is important to understand the 'true' on- and off-targets of chemical probes to appropriately interpret the observed phenotypes (pillar 4) and biochemical mechanisms (pillar 3). Only with in-depth molecular mode-of-action studies can one gain high confidence in new mechanisms of therapeutic intervention. Arguably, the selectivity criteria for chemical biology probes (Fig. 2) should be more stringent than those for drug candidates, where 'safe' promiscuity may be a desirable property to drive efficacy. Focused selectivity profiling against potential specific offtargets can also be facilitated by considering the known biological targets of small molecules that are structurally similar to the chemical probe. However, even with stringent biochemical selectivity criteria, it is always possible (and indeed likely) that a given probe has unknown off-target activity. Therefore, we also advocate the use of a structurally orthogonal chemical probe to enable cross-validation studies and the identification of an inactive close analog for use as a negative control (for example, an inactive enantiomer; Fig. 2). 
Recent advances in chemical proteomics provide the opportunity to better understand on-target and off-target engagement of small-molecule tools under physiologically relevant conditions. For example, in the kinase field, there are a number of platform technologies to assess the affinity and selectivity of small molecules in the lysate of a relevant cell. Resin immobilization of promiscuous kinase inhibitors enables the enrichment and analysis of a large proportion of the kinome, and dose-related inhibition of protein binding can be measured using quantitative MS-based proteomics ${ }^{7,8}$. Similarly, covalent ATP and ADP probes that react with conserved lysines in the ATP-binding sites of kinases can be used in competitive proteomic studies with a candidate probe or drug to determine target engagement and selectivity in a more physiological context ${ }^{9}$. Decreased cost and improved throughput of these assays have revolutionized the assessment of kinase selectivity.

Once an appropriate tool has been identified, it is worthwhile to create a bespoke chemical proteomics probe on the basis of that specific tool's chemical structure. This provides an orthogonal method to assess selectivity and target engagement in a cell and also allows an unbiased proteomic assessment (as illustrated in example below). When generating affinity probes for proteomics, it is preferable to link the molecule via several attachment points to the resin so that multiple interacting domains can be sampled in the cell lysate in an unbiased manner (as on- and off-target SARs may not be the same).

Although using the lysate of the cell of interest in determining selectivity and target engagement is a good beginning, the ultimate aim must be to make these measurements on the most relevant system-intact cells and tissues or even an in vivo model. Surprisingly, biotinylated probes can sometimes permeate cells, allowing target engagement to be assessed. For example, the application of a biotinylated probe in intact replicon cells, followed by lysis and streptavidin ELISA, was necessary to confirm the hepatitis $\mathrm{C}$ virus-encoded protein NS5A as the relevant target of replication inhibitors in an antiviral phenotypic screen ${ }^{10,11}$. In contrast, immobilization of the probe and the use of either cell lysate or recombinant NS5A failed to isolate the protein, thus illustrating the context dependence of target engagement ${ }^{10}$. Additionally, highly lipophilic fluorescently tagged inhibitors (1; Fig. $\mathbf{3}$ ) did not localize together with the NS5A protein in replicon imaging experiments, but when a 'silent' azide reporter was used instead to obtain a more relevant picture of their subcellular distribution (via subsequent 'click' tagging of the dye), complete colocalization with NS5A was observed (2; Fig. 3 $)^{12}$. These studies highlight the need for medicinal chemistry design and an appreciation of physicochemical properties of a compound to ensure viable probes are created to report on relevant intracellular interactions.

Irreversible inhibition modalities can often provide exquisitely selective chemical tools that can be subsequently furnished with additional functionalities to report on target engagement. For example, one approach has used a fluoromethylketonecontaining probe (3) to target a specific cysteine residue in the ribosomal RSK1/2 kinase active site, leading to very specific target modulation ${ }^{13}$. Moreover, by installing an alkyne click handle into the molecule (enabling subsequent biotinylation), a selective probe for isolation and enrichment of RSK was created (4) that, when applied in cells, led to further biochemical understanding of $\mathrm{RSK}^{14}$. Similar approaches have been used to quantify target occupancy (pillar 2) of irreversible BTK inhibitors (such as 5) using functional derivatives (6), allowing more accurate assessment of pharmacodynamic-pharmacokinetic correlations in early clinical trials that enabled efficacious dose projections ${ }^{15,16}$.

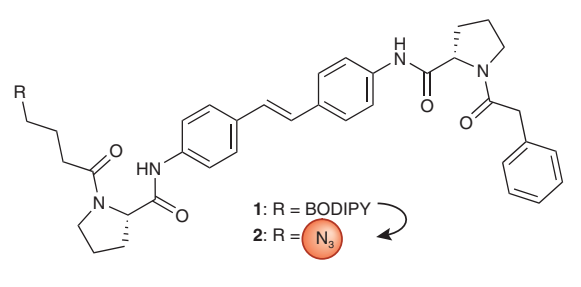

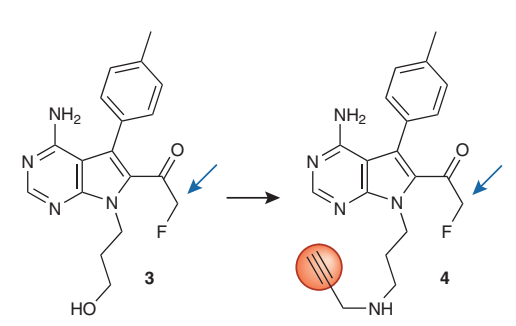

Serine hydrolase drug discovery has also been substantially augmented through the application of covalent inhibitors ${ }^{17}$. For example, the urea 7 was designed to react selectively with fatty acid amide hydrolase (FAAH) relative to other family members, which was confirmed using activity-based proteomic profiling (pillar 2$)^{18}$. In vivo dosing with probe $\mathbf{8}$, a clickable derivative of 7 , followed by tissue isolation, subsequent click reaction with rhodamine-azide and gel imaging confirmed the probe's exquisite selectivity for FAAH. In a similar manner, irreversible inhibitors bearing fluorescent or clickable tags have also been used to assess the target engagement of reversible inhibitors for their target proteins. In this case, time-dependent displacement of the reversible probe by the covalent reporter may underestimate the 'true' target occupancy, and therefore careful optimization of the experimental protocol is required ${ }^{19,20}$.

\section{Pillars 3 and 4}

A detailed discussion of pillars 3 (expression of functional pharmacology) and 4 (expression of a relevant phenotype) is beyond the scope of this commentary. However, advances in chemical biology should enable greater emphasis on screening specific pharmacological and phenotypic effects in the most relevant cellbased systems that can be obtained. Patient-<smiles>CC(C)(C)CCCCOc1ccc(Oc2cccc(C=C3CCN(C(=O)Nc4cccnn4)CC3)c2)nc1</smiles>

Figure 3 | Examples of selective chemical tools converted to functional probes to report on target engagement and distribution. Probes and their biological targets: 1 and 2 (NS5A inhibitors); 3 and 4 (irreversible RSK inhibitors); 5 and 6 (irreversible BTK inhibitors); and 7 and 8 (irreversible FAAH inhibitors). Arrows indicate the protein-reactive warhead, and red circles highlight clickable handles for subsequent attachment of fluorescent dyes or biotin reporters. 
derived cells and tissues are often the best system for translational pharmacology, particularly as these can capture pathophysiological post-translational modifications that can be lacking in simpler cell line assays or biochemical experiments using recombinant proteins. Equally important is the link between phenotypic perturbation and pathology, as many measurable biomarkers (that facilitate assay development) could simply be diseaserelated 'observations' that do not have a key causal role. In these cases, chemical probes may be found that reverse a particular phenotype but have no impact on rectifying the disease. The challenge is to unearth these possibilities in preclinical research rather than in late-stage clinical trials.

Using mammalian models to provide a disease-relevant pillar 4 can be expensive and time consuming; typically, only a small number of advanced leads can progress to such studies. One approach to circumvent these limitations is to screen molecules in primitive yet accessible organisms such as zebrafish. Zebrafish embryos are generally permeable to small molecules, thus presenting many advantages for highthroughput in vivo developmental analysis. Moreover, the size and transparency of zebrafish facilitates screening for phenotypes such as tissue development, remodeling and morphological change ${ }^{21}$.

Target validation in zebrafish comes with its own set of challenges. Relationships between physicochemical properties and zebrafish exposure (pillar 1) remain underexplored, although some studies are helpful ${ }^{22,23}$. For example, a compound with low lipophilicity $(\log \mathrm{P}<1)$ will most likely have substantially reduced absorption in zebrafish, whereas lipophilic basic compounds have higher uptake, as expected for oral drugs. Nevertheless, if permeability is a major hurdle for an otherwise desirable probe, a different route of administration can also be explored, such as microinjection ${ }^{24}$. Additionally, secondary assays must be used to rule out phenotypic outcomes masked or altered by general compound toxicity or through effects on $\beta$-catenin.

In the above sections, we have shared our perspectives on how the four pillars may be a useful framework for approaching target validation using highquality chemical tools. We would now like to illustrate these concepts through a discussion of chemical probes in the BET bromodomain field.

\section{BET bromodomain probes}

Over recent years there has been an explosion of interest in small molecules that inhibit the BET family of bromodomains (Brd2, Brd3, Brd4 and BrdT). This field was initiated by two landmark papers that described the small-molecule BET inhibitor probes (+)-JQ1 (9; Fig. 4) ${ }^{26}$ and I-BET (10; GSK525762A; Fig. 4) ${ }^{27}$. These structurally related probes were shown to inhibit protein-protein interactions between the BET bromodomains and $\mathrm{N}$-acetyl lysine residues on histones and thereby regulate gene expression. BET inhibition suppressed transcription of proinflammatory genes in models of acute inflammation and inhibited tumor cell proliferation in assays for NUT midline carcinoma (which is driven by a BRD4-NUT oncogenic fusion). This breakthrough research has inspired scientists across the globe to further explore BET bromodomain biology, the broader therapeutic potential of BET inhibition and the role of other bromodomains beyond the BET family. This work has also led to the initiation of BET inhibitor drug discovery programs within the industrial sector, with GSK525762 reportedly now in clinical trials for NUT midline carcinoma. The BET inhibitor story provides a nice demonstration of the power of chemical probes in opening up new areas of biology and target opportunity. It also illustrates the power of the holistic approach to interrogating target biology advocated in this commentary and the concepts captured by the four pillars framework. The pioneering work that identified $\mathbf{1 0}$ provides a case study in this context and will now be discussed by way of illustration ${ }^{28}$.

The discovery of BET family bromodomain inhibitor $\mathbf{1 0}$ began with a cell-based phenotypic screen, seeking small molecules that upregulated apolipoprotein A1 (ApoA1) expression. ApoA1 is associated with antiinflammatory activity and protection from atherosclerosis; however, no direct molecular mechanisms for modulating ApoA1 had been described at the onset of the study. Chung et al. ${ }^{28}$ devised a robust high-throughput phenotypic assay based on a luciferase reporter system in HepG2 cells (pillar 4), which led to the discovery of benzodiazepine 11 (Fig. 4) that upregulated ApoA1 expression with a half-maximum
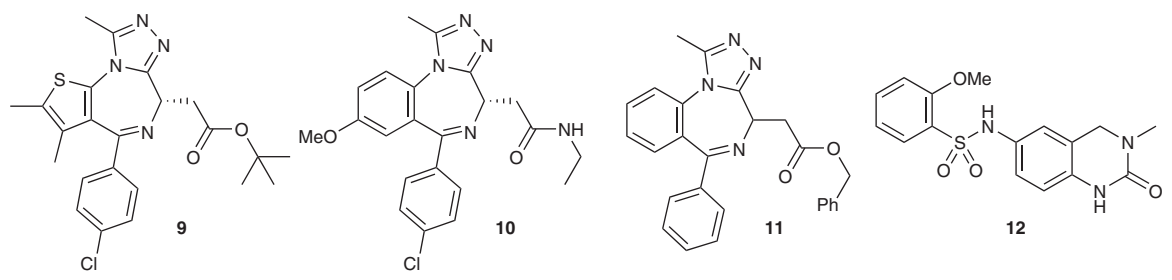

Figure 4 | Selective BET bromodomain chemical probes. effective concentration of $440 \mathrm{nM}$. Medicinal chemistry optimization of the physical properties in this template led to $\mathbf{1 0}$, which had similar activity to $\mathbf{1 1}$ in the ApoA1 assay. Chung et al. ${ }^{28}$ then conducted a master class in chemical biology to discover the BET proteins as the molecular targets of 10. First, they showed that the enantiomer of $\mathbf{1 0}$ was inactive in the phenotypic assay, thus providing a perfect control for nonspecific activity (Fig. 2). Chemical proteomic methods using a pull-down reagent developed through conjugation of $\mathbf{1 0}$ to a ReactiGel matrix were then used to seek the molecular target of $\mathbf{1 0}$ (ref. 28). Application of this reagent in HepG2 lysates pulled down a distinct proteome that could be competed away by 10 itself (a pillar 2 technology). Control studies using a ReactiGel matrix alone or a control pull-down reagent derived from the inactive antipode of $\mathbf{1 0}$ did not capture these proteins. LC/MS/ MS identified the bromodomain proteins $\operatorname{Brd} 2, \operatorname{Brd} 3$ and Brd4 as the putative targets $^{28}$. Cloning and expression of the isolated bromodomains and a combination of biophysical techniques, including $\mathrm{X}$-ray crystallography, allowed Chung et al..$^{28}$ to show that $\mathbf{1 0}$ bound distinct modules within the BET bromodomains that recognize acetylated lysine motifs on histones. This work demonstrated that $\mathbf{1 0}$ most likely inhibited BET bromodomain binding to chromatin, which in turn directly regulated the expression of associated genes. This finding was further supported by broader pharmacological profiling of $\mathbf{1 0}$ (pillar 3) that indicated the probe had negligible activity beyond binding the BET family. Furthermore, extensive SAR analyses showed that BET inhibitory activity strongly correlated with whole-cell potency for ApoA1 upregulation, further supporting this target-phenotype association in cells (pillars 1 and 4). Finally, orthogonal validation studies were conducted using RNAi knockdown experiments in HepG2 cells, which showed that siRNA for Brd4 recapitulated the ApoA1 upregulation phenotype. Overall, through this deep and unbiased chemical biology program, Chung et al. ${ }^{28}$ were able 
to confidently link the direct inhibition of BET bromodomains with the ApoA1 upregulation phenotype and validate $\mathbf{1 0}$ as a high-quality chemical probe for the inhibition of BET bromodomains in cells.

In the paper describing the discovery of (+)-JQ1, the selectivity of this probe for the BET family was also very well characterized, and the antipode of $\mathbf{9}$ was similarly used as an inactive control for phenotypic screening studies ${ }^{26}$. Interestingly, Filippakopoulos et al. ${ }^{26}$ used a fluorescence recovery after photobleaching (FRAP) assay using GFPtagged Brd4 to demonstrate that 9 inhibited binding of Brd4 to chromatin in a cellular context (pillar 3). They also validated the potential of BET inhibitors in treating NUT midline carcinoma using a highly relevant phenotypic assay that used cells taken from patients (pillar 4).

The availability of (+)-JQ1 and I-BET has recently been complemented by the publication of PFI-1 (12) from our own laboratories ${ }^{29}$. Importantly, PFI-1 is structurally orthogonal to $\mathbf{9}$ and $\mathbf{1 0}$ and thus can serve as a valuable tool for cross-validating the target-phenotype associations for BET inhibition (Fig. 2). Altogether, the BET inhibitor field is now enabled by a diverse set of chemical probes, inactive controls, siRNA tools and chemical proteomic reagents that allow a deep interrogation of the biology of the BET protein family in an unbiased way ${ }^{30}$.

The BET bromodomain case history illustrates the importance of providing all four pillars and the considerable investments required to understand the link between target modulation and potential therapeutic effects. Equally, it also shows that the order in which the pillars are built is not necessarily important and that the entry point can vary depending on the nature of the discovery program. In this case, pillar 4 (phenotype) led to pillar 2 (target engagement), which supplied pillar 1 (exposure) and was followed by pillar 3 (pharmacology).

\section{Outlook}

As we and others have argued, the biggest challenge faced in pharmaceutical research and development is to reduce phase 2 attrition rates by identifying molecular targets that have a high probability of influencing disease ${ }^{31}$. In a preclinical setting, disease-relevant phenotypic assays that instill confidence in their translation to the clinic are essential. Small-molecule probes are powerful tools in helping identify and/or validate potential new molecular targets that drive these phenotypic responses. However, drawing conclusions on target-phenotype relationships using a single small-molecule probe in isolation can be a risky endeavor. The scientific literature is unfortunately rich with examples of claims based on single entities, and the type of deeper interrogation of target-phenotype relationships through a diverse chemical toolbox, as illustrated in the BET inhibitor field, is the exception rather than the rule. We hope the four pillars concept discussed here can serve as a useful framework to consider target validation in a preclinical setting and help in the identification of targets that are more likely to deliver future new medicines. We also suggest that the framework described herein can also be applied in preclinical animal models to build confidence in translational pharmacology and to other modalities additional to small-molecule probes, such as peptides, proteins, RNAi and antisense oligonucleotides.

Mark E. Bunnage, Eugene L. Piatnitski Chekler and Lyn H. Jones are in Worldwide Medicinal Chemistry at Pfizer, Cambridge, Massachusetts, USA. e-mail:mark.bunnage@pizer.com, eugene.chekler@pfizer.com orlyn.jones@pfizer.com
References

1. Frye, S.V. Nat. Chem. Biol. 6, 159-161 (2010).

2. Workman, P. \& Collins, I. Chem. Biol. 17, 561-577 (2010).

3. Morgan, P. et al. Drug Discov. Today 17, 419-424 (2012).

4. Sarker, D. \& Workman, P. Adv. Cancer Res. 96, 213-268 (2007).

5. Lipinski, C.A., Lombardo, F., Dominy, B.W. \& Feeney, P.J. $A d v$. Drug Deliv. Rev. 46, 3-26 (2001).

6. Zheng, N., Tsai, H.N., Zhang, X. \& Rosania, G.R. Mol. Pharm. 8 1619-1628 (2011)

7. Bantscheff, M. et al. Nat. Biotechnol. 25, 1035-1044 (2007).

8. Wissing, J. et al. Mol. Cell. Proteomics 6, 537-547 (2007).

9. Patricelli, M.P. et al. Chem. Biol. 18, 699-710 (2011).

10. Targett-Adams, P. et al. J. Virol. 85, 6353-6368 (2011).

11. Gao, M. et al. Nature 465, 96-100 (2010).

12. Jones, L.H. et al. J. Chem. Biol. 4, 49-53 (2011).

13. Cohen, M.S., Zhang, C., Shokat, K.M. \& Taunton, J. Science 308, 1318-1321 (2005)

14. Cohen, M.S., Hadjivassiliou, H. \& Taunton, J. Nat. Chem. Biol. 3, 156-160 (2007).

15. Honigberg, L.A. et al. Proc. Natl. Acad. Sci. USA 107, 13075 13080 (2010).

16. Westlin, W.F. et al. Cancer Res. doi:10.1158/1538-7445.AM2012-1745 (15 April 2012).

17. Simon, G.M. \& Cravatt, B.F. J. Biol. Chem. 285, 11051-11055 (2010).

18. Ahn, K. et al. J. Pharmacol. Exp. Ther. 338, 114-124 (2011).

19. Adibekian, A. et al. J. Am. Chem. Soc. 134, 10345-10348 (2012).

20. Barkovich, K.J. et al. Cancer Discov. 2, 450-457 (2012).

21. Peterson, R.T. \& Fishman, M.C. Methods Cell Biol. 105, 525-541 (2011).

22. Padilla, S. et al. Reprod. Toxicol. 33, 174-187 (2012).

23. Sachidanandan, C., Yeh, J.R., Peterson, Q.P. \& Peterson, R.T. PLoS One 3, e1947 (2008).

24. Milan, D.J., Peterson, T.A., Ruskin, J.N., Peterson, R.T. \& MacRae, C.A. Circulation 107, 1355-1358 (2003).

25. Zhang, M., Zhang, J., Lin, S.-C. \& Meng, A. Development 139, 2009-2019 (2012)

26. Filippakopoulos, P. et al. Nature 468, 1067-1073 (2010).

27. Nicodeme, E. et al. Nature 468, 1119-1123 (2010).

28. Chung, C.-W. et al. J. Med. Chem. 54, 3827-3838 (2011).

29. Fish, P.V. et al. J. Med. Chem. 55, 9831-9837 (2012).

30. Weiss, W.A., Taylor, S.S. \& Shokat, K.M. Nat. Chem. Biol. 3, 739-744 (2007).

31. Bunnage, M.E. Nat. Chem. Biol. 7, 335-339 (2011).

\section{Acknowledgments}

We thank many colleagues at Pfizer for useful discussions on this topic. We also thank S. Frye (University of North Carolina), J. Bradner (Harvard University, Dana-Farber Cancer Institute), J. Blagg (Institute of Cancer Research, UK), A. Edwards (University of Toronto), C. Bountra (University of Oxford) and P. Brennan (University of Oxford) for their helpful feedback.

Competing financial interests

The authors declare competing financial interests: details accompany the online version of the paper. 